\title{
Standwaage, Squat und Co.
}

\section{Neue Serie: Übung des Monats}

Physiotherapeuten haben einen riesigen Schatz an Übungen. Diejenigen, die für Reha und

Therapie wichtig sind, schauen sich die Sportphysiotherapeuten Matthias Keller und Volker Hacker in der neuen physiopraxis-Serie „Übung des Monats“ genauer an. Für welche Patienten eignet sich die Übung? Wann setze ich sie ein? Und wie erkenne und korrigiere ich häufige Fehler?

Matthias Keller und Volker Hacker sind Physiotherapeuten und Dozenten im OSINSTITUT. Ihr therapeutischer Schwerpunkt liegt in der aktiven Therapie, der muskuloskelettalen Reha und dem funktionellen Training. In den nächsten Ausgaben von physiopraxis präsentieren sie die Rubrik „Übung des Monats“. Dabei stellen sie nicht nur wichtige Übungen vor, sondern gehen auch auf die Feinheiten ein. Neben einer optimalen Anleitung für die Übung geben sie einen Überblick, welche Fehler häufig auftreten und wo sich die Übungen am besten einsetzen lassen. Alle Übungen sind auch als Video abrufbar (○ S. 28).

\begin{tabular}{ll} 
Regression & Progression \\
\hline$\rightarrow$ kürzerer Hebel & $\rightarrow$ längerer Hebel \\
\hline$\rightarrow$ bilateral & $\rightarrow$ kleinere Unterstützungsfläche \\
\hline$\rightarrow$ kleinere Gewichte/Widerstand & $\rightarrow$ größere Gewichte/Widerstand \\
\hline$\rightarrow$ langsamere Ausführung & $\rightarrow$ schnellere Ausführung \\
\hline$\rightarrow$ stabile Unterstützungsfläche & $\rightarrow$ instabile Unterstützungsfläche \\
\hline$\rightarrow$ einfache Muster & $\rightarrow$ komplexe Muster \\
\hline$\rightarrow$ isolierte Bewegungen & $\rightarrow$ integrierte Bewegungen \\
\hline$\rightarrow$ geringerer Umfang & $\rightarrow$ höherer Umfang \\
\hline$\rightarrow$ längere Pausen & $\rightarrow$ kürzere Pausen \\
\hline
\end{tabular}

TAB. 1 Eigenschaften progredienter und regressiver Übungsmodifikationen
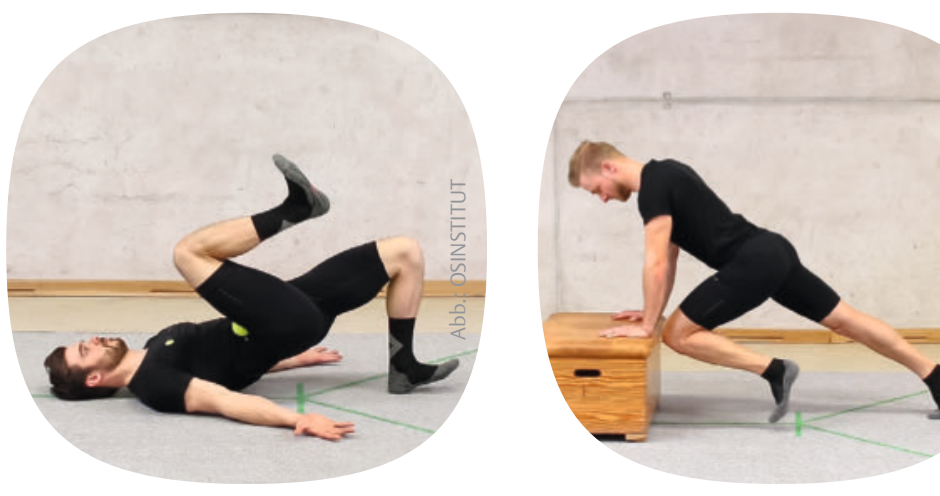

Trainingspläne zu erstellen und dabei passende Übungen auszuwählen, gehört zur Domäne von Physiotherapeuten und Sportwissenschaftlern. Immer häufiger kommt es jedoch vor, dass Ärzte ihren Patienten Broschüren mit Übungen an die Hand geben und diese empfehlen, anstatt aktive Therapie zu verordnen. Das so wichtige Anleiten und Coachen einer Übung findet nicht statt. Für das motorische Lernen und um Bewegungsmuster zu verbessern, ist dies aber elementar. Eine Broschüre gibt dem Patienten kein Feedback und zeigt auch keine Alternativen, wenn eine Übung nicht funktioniert.

Bei der Erstellung eines Trainingsplans ist es wichtig, verschiedene Aspekte zu bedenken. Es steht nicht nur die Auswahl der Übung, sondern auch das richtige Coaching im Vordergrund. Dabei geht es vor allem darum, eine Übung so anzuleiten, dass der Patient sie korrekt ausführt. Progressionen und Regressionen helfen Physiotherapeuten dabei, eine Basisübung so zu steuern, dass sie schwerer oder einfacher in der Ausführung wird oder einen völlig neuen Charakter bekommt. Veränderungen an einer Übung, die keinen neuen Effekt hervorrufen, sondern nur eine Version der eigentlichen Übung sind, bezeichnet man als Variation (॰ ABB. 1).

Für die Auswahl der Übung sind folgende Überlegungen wichtig:

$\rightarrow$ Was möchte ich mit der Übung erreichen?

$\rightarrow$ Stimmt die Übung mit dem Patientenziel überein?

$\rightarrow$ Passt die Übung zum Anforderungsprofil des Patienten?

$\rightarrow$ Lassen eine mögliche Pathologie und das Wundheilungsstadium die Ausführung der Übung zu? 


\section{Therapie | Übung des Monats}

\section{Die Standwaage}

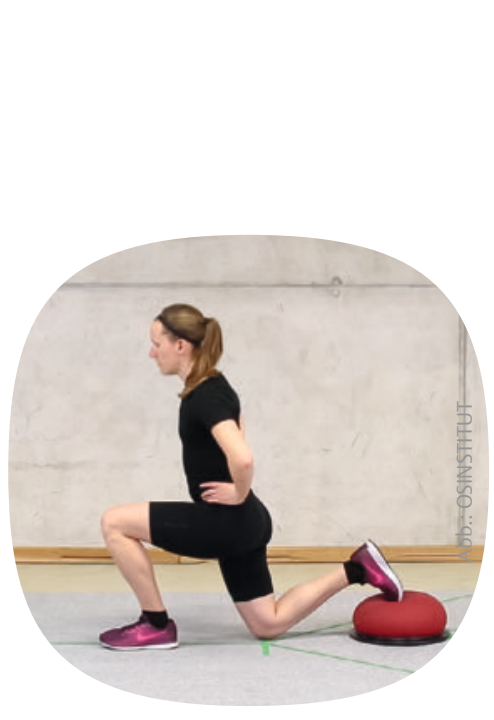

Den Beginn unserer Serie „Übung des Monats" macht die Standwaage.

Lesen Sie mehr ab S. 28!
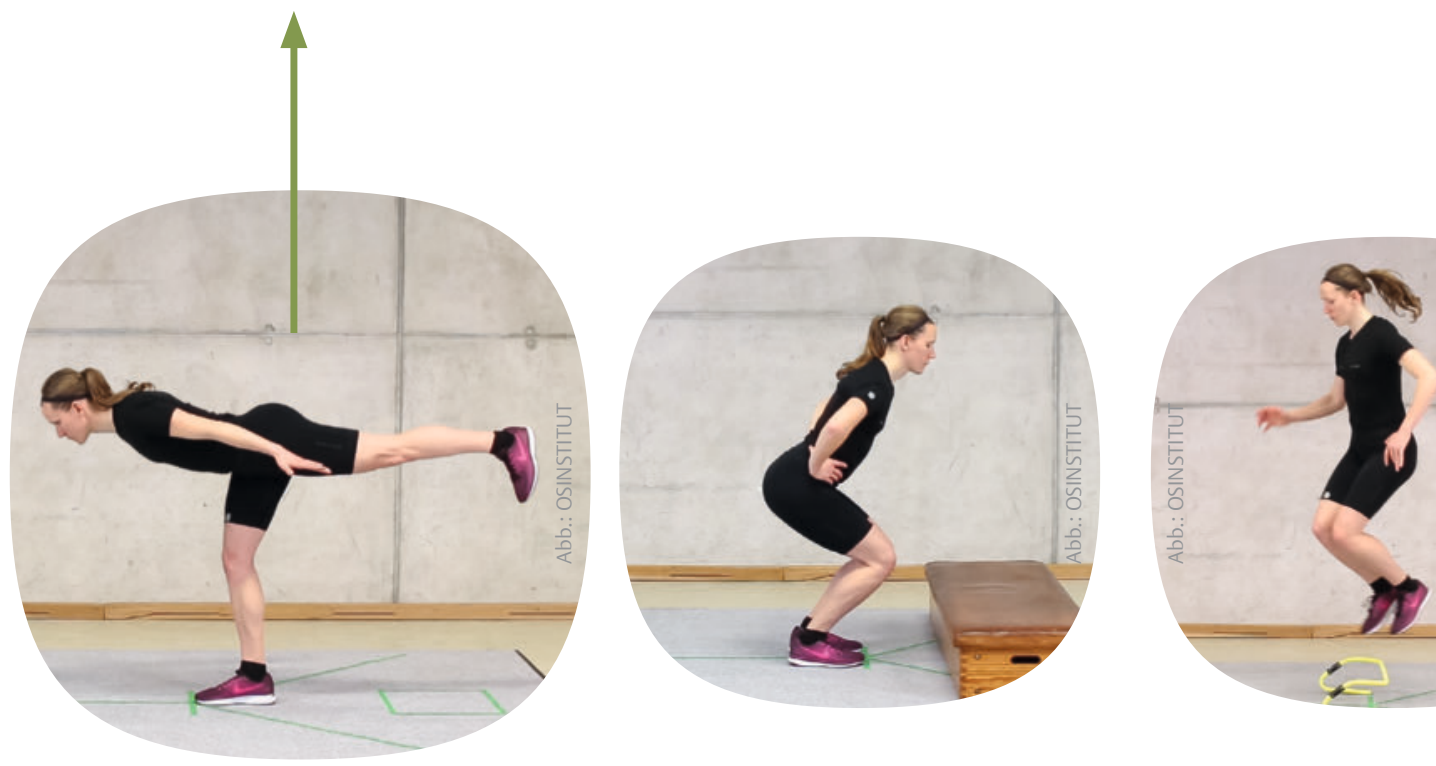

$\rightarrow$ Sind die logistischen Voraussetzungen vorhanden (Equipment, Platz usw.)?

Die Basisübung $\rightarrow$ Die Basisübung kann als „Reinform“ eines Manche Übungen fallen dem Patienten auch schwerer, wenn er sie sehr langsam ausführen soll. Damit bleiben die Auswahl und das Coaching einer Übung immer für jeden Patienten individuell.

Matthias Keller und Volker Hacker Bewegungsmusters bezeichnet werden. Sie wird im Idealfall einfach vom Therapeuten angeleitet und vom Patienten umgesetzt. In der Regel ist dafür kein oder nur wenig Equipment erforderlich. Die richtige Ausführung spielt dabei eine ebenso große Rolle wie die Auswahl der Übung. Die Möglichkeiten, eine Übung zu korrigieren, wenn ein Patient diese nicht ganz korrekt umsetzt, sind visuelles, akustisches und taktiles Feedback.

Regression und Progression $\rightarrow$ Therapeuten können eine Übung durch viele Stellschrauben verändern und wie bei einem Mischpult mit Reglern sehr fein justieren. Dadurch kann sich die Basisübung so verändern, dass die Ausführung für den Patienten einfacher oder schwerer wird. Hinsichtlich dieser Übungsmodifikationen kann man grundsätzlich von einer methodischen Reihe sprechen (॰ TAB. 1). Diese schematische Einteilung lässt sich jedoch nicht auf jede Übung und jeden Patienten unreflektiert übertragen. So kann beispielsweise ein leichtes Zusatzgewicht als Führungswiderstand auch eine Hilfe für den Patienten sein, die Übung durchzuführen.

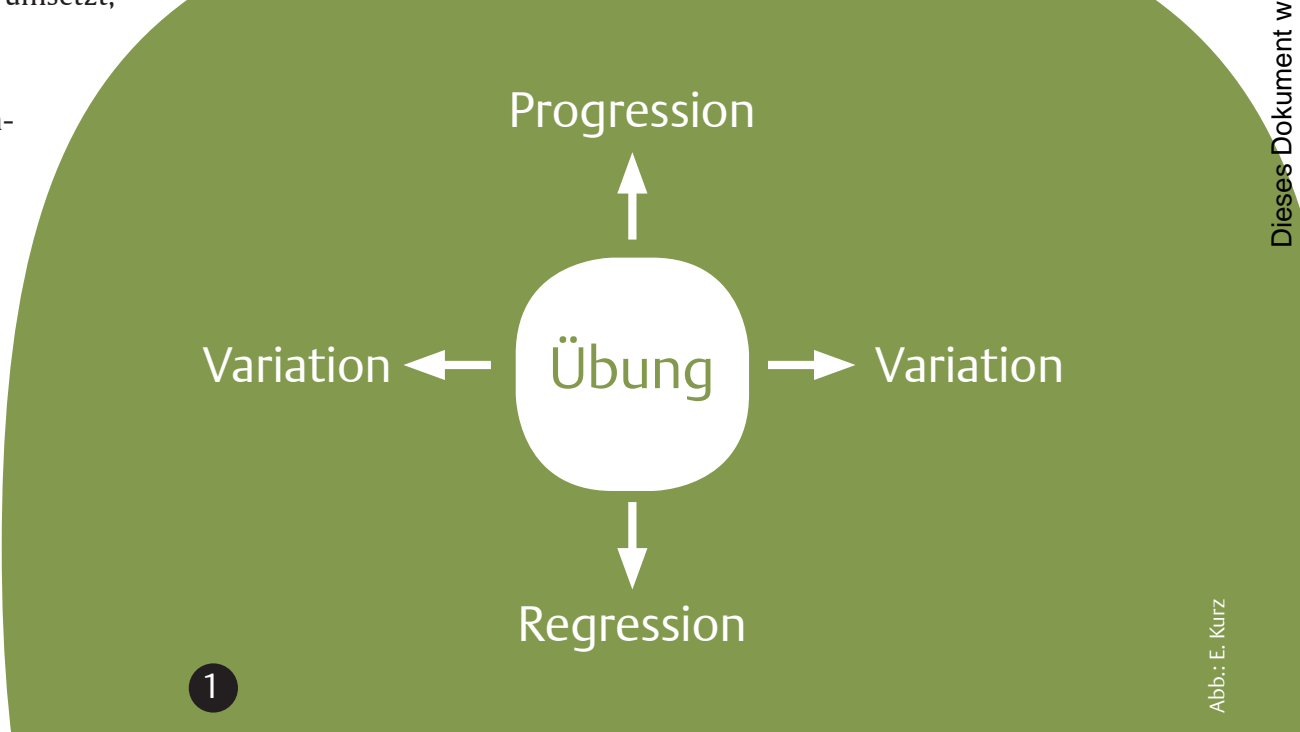




\section{Übung des Monats}

\section{Die Standwaage}

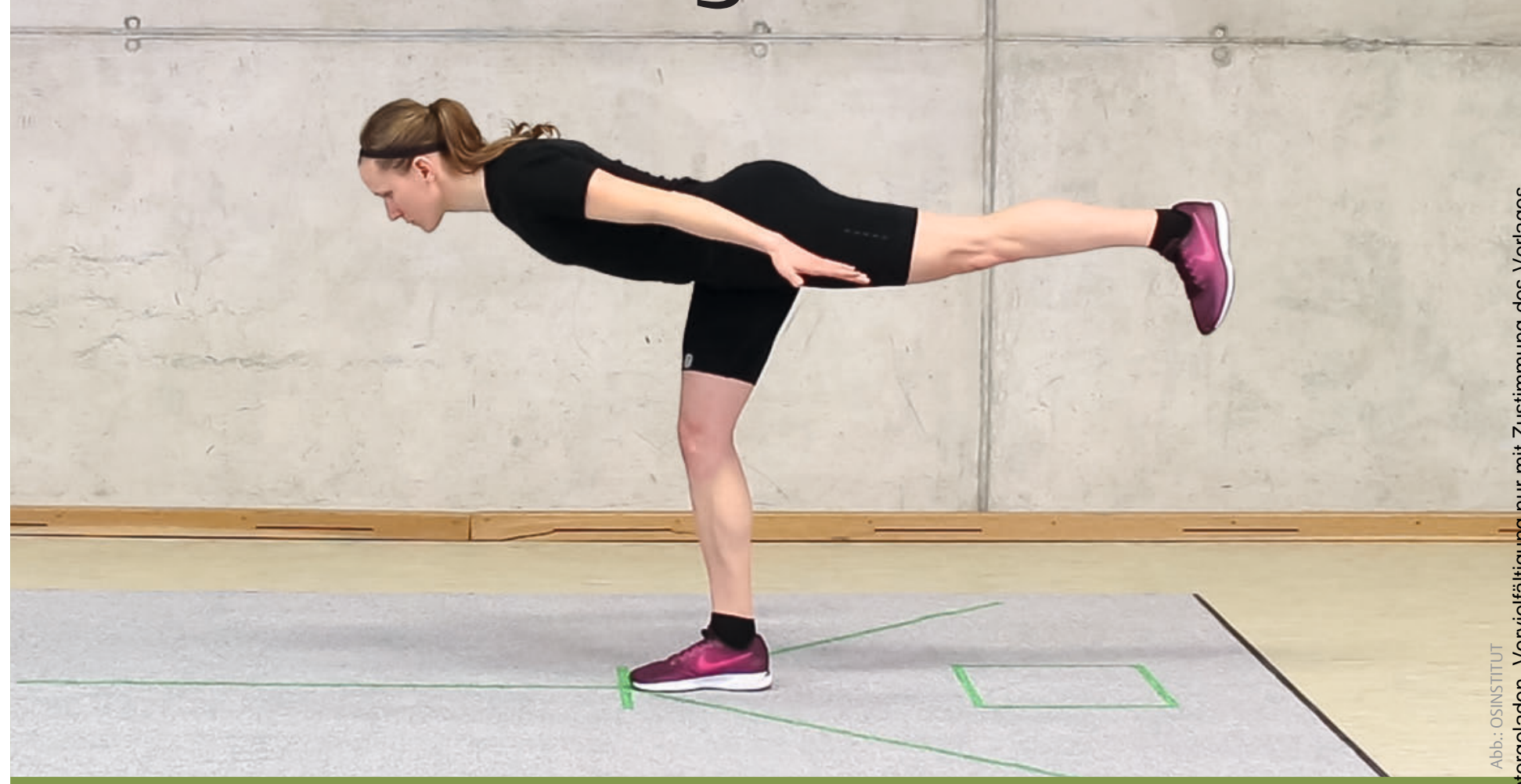

Die Standwaage zählt zu den bekanntesten Übungen im Einbeinstand. Sie fordert Kraft, Mobilität und motorische Kontrolle und wird im Yoga, Functional Training und in der muskuloskelettalen Reha eingesetzt.

\section{Coaching Cues}

Phase 1 Kniehub: „Stelle dich auf dein rechtes Bein. Beuge gleichzeitig Knie und Hüfte deines linken Beins, bis die Hüfte maximal gebeugt ist, ohne eine Bewegung in Becken und Wirbelsäule zuzulassen."

Phase 2 Standwaage: „Bewege aus dieser Position deinen Oberkörper mit neutraler Wirbelsäule über eine Beugung der rechten Hüfte so weit nach vorne, bis er sich möglichst parallel zur Unterlage befindet. Gleichzeitig führst du dein linkes Bein (Spielbein) nach hinten, bis es in der Endstellung in Knie und Hüfte gestreckt ist und eine Gerade mit dem Oberkörper bildet. Halte während der ganzen Bewegung das Kniegelenk deines rechten Beines (Standbein) in einer leichten Beugung (Entriegelung Kniegelenk). “

\section{Die Übung im Video}

Auf www.osinstitut.de bzw.

unter dem QR-Code finden Sie unsere

„Übungen des Monats“ auch im Video,

inklusive Beispielen von häufigen Fehlern

und wie man sie in der Therapie korrigiert.

\section{Background}

Die Standwaage ist auch als Viravadrasana III, Krieger III, Inverted Hamstring, Single Leg Deadlift und Single Leg Romanian Deadlift bekannt. Es handelt sich um eine Hip-Hinge-(Hüftscharnier-) Bewegung - der Drehpunkt ist immer das Hüftgelenk. In der Wirbelsäule und im Kniegelenk findet keine aktive Bewegung statt. Dadurch eignet sich die Übung sehr gut, um die statische Kontrolle zu verbessern, und ist relativ früh nach Knieverletzungen einsetzbar. Auch bei Dysfunktionen der motorischen Kontrolle (Control Impairment) an der LWS hat die Übung eine große Relevanz, da der Patient seine Lordose während der Bewegung immer kontrollieren muss. Durch die einbeinige Ausgangsstellung trainiert man die posturale Kontrolle. Da die Standwaage ein umgekehrter Active Straight Leg Raise ist, kommt in der Endposition die dorsale Kette auf Länge, sodass die Hamstrings und auch der $\mathrm{N}$. ischiadicus mobilisiert werden.

Die Standwaage vereint motorische Kontrolle und Mobilisation und wird daher im Sport gerne innerhalb des Warm-ups eingesetzt. Dabei ist sie fester Bestandteil innerhalb der dynamischen Beweglichkeit und den Movement Preps. Um eine Standwaage ausführen zu können, muss der Patient auf einem Bein stehen können, und eine Vollbelastung ist als Voraussetzung erforderlich. Außerdem ist eine gewisse Mobilität in der dorsalen Kette notwendig. 


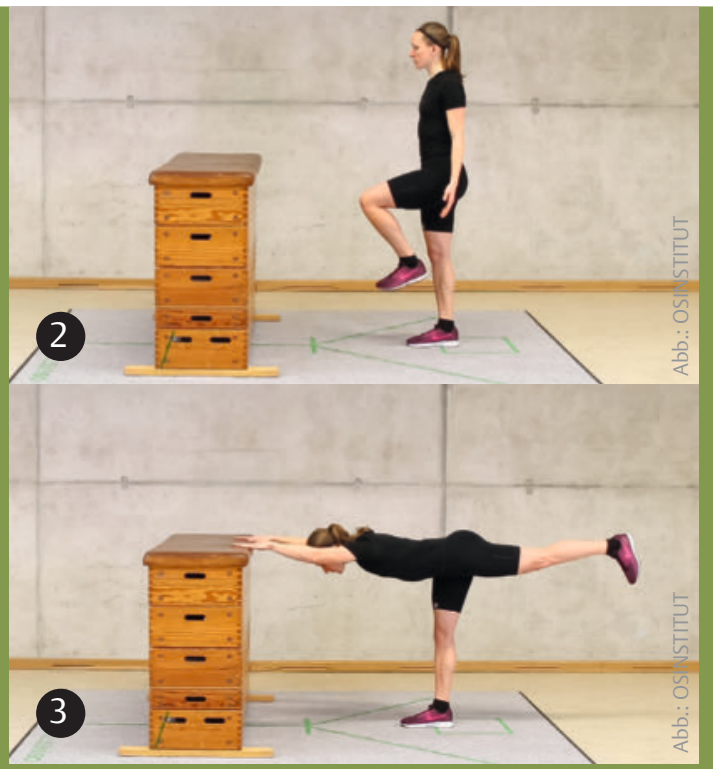

Regression

Gerade für unsichere und ältere Menschen ist die Standwaage eine koordinative Herausforderung. Oft kann die Übung aufgrund eines unzulänglichen Gleichgewichtssinns nicht über das volle Bewegungsausmaß durchgeführt werden.

Eine geeignete Regression für diese Gruppe ist es, sich vor einen Gegenstand zu stellen, auf den sie sich zubewegen können. In der

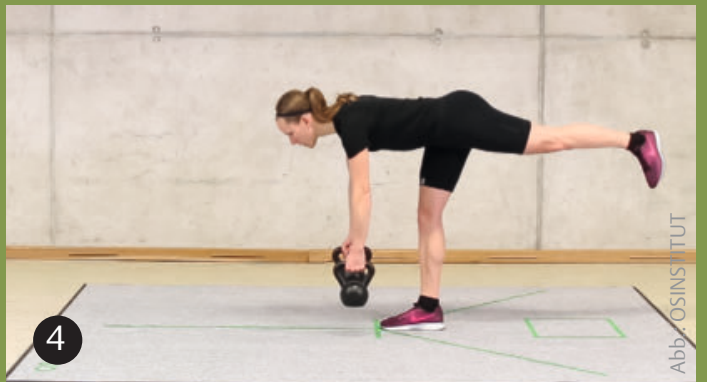

\section{Progression}

Um bei der Standwaage die Komponente Kraft zu betonen, kann der Trainierende die Übung mit Zusatzgewichten durchführen. Des Weiteren kann er in der Endposition beispielsweise eine Ruderbewegung mit Kettlebells machen.

Dadurch wird nicht nur der koordinative Anspruch der Übung größer, auch der Oberkörper wird hinsichtlich einer ziehenden Übung trainiert. Die Höhe des Gewichts und die Umfänge bestimmen dabei die Intensität.

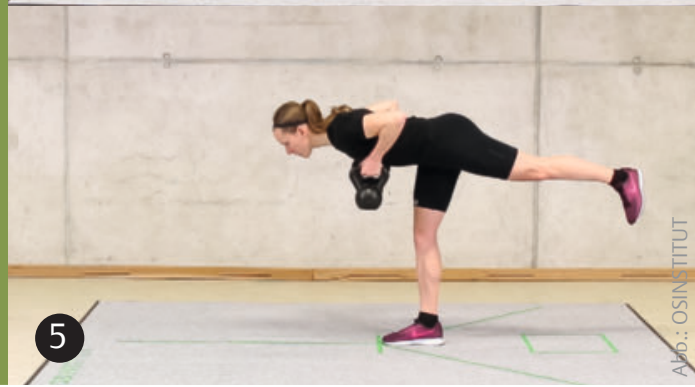

\section{lichkeiten}

Fehler 1: Der Trainierende kann die geforderte Kniegelenksstellung des Standbeins während der Übung nicht stabilisieren. Er überstreckt das Kniegelenk oder beugt es vermehrt.

Korrektur: Er hält sich in der Endposition fest und beugt und streckt isoliert nur das Kniegelenk, bis der korrekte Gelenkwinkel erarbeitet ist.

Fehler 2: Der Trainierende hält die neutrale Ausrichtung der Wirbelsäule in den Bewegungsphasen nicht. In der Kniehubphase zieht die endgradige Beugung des Hüftgelenks des Spielbeines weiterlaufend das Becken nach posterior und somit die LWS in Flexion.
Korrektur: Der Trainierende hält sich als Feedback für eine neutrale Wirbelsäulenaufrichtung einen Stab in den Rücken. Die Kontaktpunkte sind Hinterhaupt, Scheitelpunkt der BWS-Kyphose und Kreuzbein.

Fehler 3: Der Patient dreht das Becken auf der Spielbeinseite über eine Außenrotation des Hüftgelenks des Standbeins auf.

Korrektur: Der Therapeut korrigiert über reaktives neuromuskuläres Training: Der Trainierende muss das Becken gegen diesen taktilen Reiz über eine aktive Hüftgelenkinnenrotation des Standbeins in die Horizontale bewegen.

\section{Autoren}

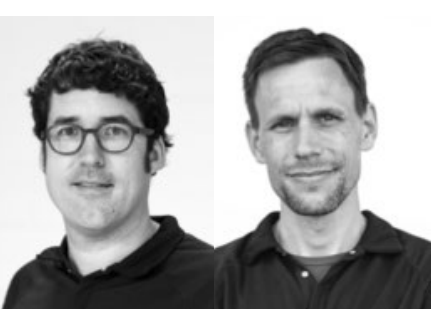

Matthias Keller ist Physiotherapeut, B.A., leitet das OSINSTITUT - Bewegung für Orthopädie und Sportmedizin und ist bei OSPHYSIO als Physiotherapeut tätig. Er arbeitet als Dozent für medizinische Trainingstherapie und ist Mitglied der Komitees „Prävention, konservative Therapie und Rehabilitation“ der Deutschen Kniegesellschaft e. V. und „Rehabilitation“ der Gesellschaft für Arthroskopie und Gelenkchirurgie. Er ist Herausgeber der Thieme Zeitschrift „Sportphysio“.

Volker Hacker ist Physiotherapeut und im Dozententeam des OSINSTITUTs. Er ist mitverantwortlich für die Konzeption der Seminare und leitet diese an verschiedenen Standorten. Bei seiner Tätigkeit bei OSPHYSIO liegen seine Schwerpunkte in der Manuellen Therapie, der Medizinischen Trainingstherapie und dem Functional Training. 